Research Article

\title{
Some Remarks on the Solution of Linearisable Second-Order Ordinary Differential Equations via Point Transformations
}

\author{
Winter Sinkala 1 (1) \\ Department of Mathematical Sciences and Computing, Faculty of Natural Sciences, Walter Sisulu University, Private Bag X1, \\ Mthatha 5117, South Africa \\ Correspondence should be addressed to Winter Sinkala; sinkalaw@gmail.com
}

Received 29 March 2020; Accepted 18 May 2020; Published 1 July 2020

Academic Editor: Ji Gao

Copyright (c) 2020 Winter Sinkala. This is an open access article distributed under the Creative Commons Attribution License, which permits unrestricted use, distribution, and reproduction in any medium, provided the original work is properly cited.

Transformations of differential equations to other equivalent equations play a central role in many routines for solving intricate equations. A class of differential equations that are particularly amenable to solution techniques based on such transformations is the class of linearisable second-order ordinary differential equations (ODEs). There are various characterisations of such ODEs. We exploit a particular characterisation and the expanded Lie group method to construct a generic solution for all linearisable second-order ODEs. The general solution of any given equation from this class is then easily obtainable from the generic solution through a point transformation constructed using only two suitably chosen symmetries of the equation. We illustrate the approach with three examples.

\section{Introduction}

Nonlinear ODEs arise in many different contexts, including as mathematical models of real-world phenomena. Analytical solutions of many such equations are often hard to find, which is why a whole range of methods have been proposed for investigating different types of nonlinear ODEs. These methods include Painlevé singularity analysis, Lie symmetry analysis, Darboux method, and the Jacobi last multiplier method (see [1] and the references therein). The Lie symmetry method, which is based on the invariance of a differential equation under a continuous group of point transformations, is widely used. Given a differential equation, Lie point symmetries of the equation can be used to perform many things on the equation including constructing explicit transformations that reduce the equation to a simpler one, when this is possible. When the simpler "target" equation is a linear equation, the problem is called the linearisation problem. The pioneering work on this, with regard to second-order ODEs, is attributed to Sophus Lie (see [2] and the references there in). Lie proved that, to be linearisable, a second-order ODE must be at most cubically semilinear, and the coefficients in it must satisfy an overdetermined system of conditions [3, 4]. A considerable amount of research has since been conducted on the linearisation problem $[2,3,5-7]$ leading, in particular, to a variety of ways of characterising linearisable second-order ODEs (see, e.g., Theorem 8 of [3]). A scaled-down version of Theorem 8 of [3] is presented here.

Theorem 1. Let us consider a scalar second-order ODE in the form

$$
y^{\prime \prime}=f\left(x, y, y^{\prime}\right) .
$$

The following statements are equivalent [3]:

(1) A scalar second-order equation (1) is linearisable via a point transformation.

(2) Equation (1) has the maximum eight-dimensional symmetry Lie algebra.

(3) Equation (1) has the cubic in derivative form 


$$
y^{\prime \prime}=A(x, y) y^{\prime 3}+B(x, y) y^{\prime 2}+C(x, y) y^{\prime}+D(x, y)
$$

with the coefficients $A$ to $D$ satisfying the two invariant conditions

$$
\begin{gathered}
3 A_{x x}+3 A_{x} C-3 A_{y} D+3 A C_{x}+C_{y y} \\
-6 A D_{y}+B C_{y}-2 B B_{x}-2 B_{x y}=0 \\
6 A_{x} D-3 B_{y} D+3 A D_{x}+B_{x x}-2 C_{x y} \\
-3 B D_{y}+3 D_{y y}+2 C C_{y}-C B_{x}=0 .
\end{gathered}
$$

(4) Equation (1) has two noncommuting symmetries $X_{1}$ and $X_{2}$ in a suitable basis with

$$
\left[X_{1}, X_{2}\right]=X_{1}, X_{1} \neq \rho(x, y) X_{2},
$$

for any nonconstant function $\rho$ such that a point change of variables $X=X(x, y), Y=Y(x, y)$ which brings $X_{1}$ and $X_{2}$ to their canonical form

$$
\begin{aligned}
& X_{1}=\frac{\partial}{\partial Y} \\
& X_{2}=X \frac{\partial}{\partial X}+Y \frac{\partial}{\partial Y},
\end{aligned}
$$

reduces equation (1) to

$X Y^{\prime \prime}=\frac{b}{3 a}+\frac{b^{3}}{27 a^{2}}+\left(1+\frac{b^{2}}{3 a}\right) Y^{\prime}+b Y^{\prime 2}+a Y^{\prime 3}$

where $a(\neq 0)$ and $b$ are constants.

In this paper, we take advantage of representation (6) to construct a proxy solution for all linearisable secondorder ODEs. We start by using the expanded Lie group method to simplify equation (6) significantly. We then construct two point transformations and use them to recover the general solution of equation (6) from the solution of the simplest second-order ODE, the free particle equation $Y^{\prime \prime}=0$. The solution of any given linearisable second-order ODE can now be recovered from the solution of equation (6) in a routine fashion via a point transformation constructed from only two symmetries of the equation. We illustrate the solution routine through three examples.

The rest of the paper is organised as follows. In Section 2 , we use the expanded Lie group method to construct a point transformation that reduces equation (6) to a simpler equation. In Section 3, we perform further reduction and map equation (6) to the free particle equation. We subsequently deduce a generic solution of all linearisable second-order ODEs. In Section 4, we illustrate through three examples how the general solution of any linearisable second-order ODE may be deduced from the constructed generic solution. We give concluding remarks in Section 5.

\section{Reduction of Equation (6) via the Expanded Lie Group Method}

The Lie symmetry method for studying differential equations, initiated by Sophus Lie in the latter part of the nineteenth century, is based on continuous groups of transformations that map solutions of a given differential equation into other solutions of the same equation. The method extends and harmonises various specialised methods for solving ODEs. There is extensive literature on the Lie symmetry method, to which we refer the interested reader to see, for example, [4, 8-13].

When we consider a continuous group of transformations acting on the expanded space of variables, which includes the equation parameters in addition to independent and dependent variables, we obtain an expanded Lie group transformation of the equation [14]. Such a group of transformations represents a particular case of the equivalence group that preserves the class of equations under study.

Let us take the parameter $b$ in equation (6) as a second independent variable. Now, consider a one-parameter $(\varepsilon)$ Lie group of point transformations in $(X, Y, b)$,

$$
\begin{aligned}
\widetilde{X} & =f(X, Y, b, \varepsilon), \\
\widetilde{Y} & =g(X, Y, b, \varepsilon), \\
\widetilde{b} & =h(b, \varepsilon),
\end{aligned}
$$

for some functions $f, g$, and $h$, with an infinitesimal generator of the form

$$
X=\xi(X, Y, b) \partial_{X}+\tau(X, Y, b) \partial_{Y}+\beta(b) \partial_{b},
$$

which leaves equation (6) invariant. Equation (6) admits (7) if $X$ satisfies the invariance requirement:

$$
\left.X^{[2]}\left\{\frac{b}{3 a}+\frac{b^{3}}{27 a^{2}}+\left(1+\frac{b^{2}}{3 a}\right) Y^{\prime}+b Y^{\prime 2}+a Y^{\prime 3}-X Y^{\prime \prime}\right\}\right|_{(6)}=0
$$

where $X^{[2]}$ is the second extension of $X$. We obtain, as a particular solution of (9), that

$$
\begin{aligned}
& \xi=\frac{1+X^{2}}{2 X} \\
& \tau=Y\left[\frac{1+X^{2}}{4 X}+\frac{1}{2}\left(1-\frac{1+X^{2}}{2 X^{2}}\right)\right]-\frac{b}{6 a X}-\frac{X}{3 a},
\end{aligned}
$$

$$
\beta=1
$$

The corresponding one-parameter $(\varepsilon)$ Lie group of transformations is

$$
\begin{aligned}
& \tilde{X}=\sqrt{e^{\varepsilon}\left(1+X^{2}\right)-1}, \\
& \tilde{Y}=\frac{e^{(\varepsilon / 2)}(3 a Y+b X)-(b+\varepsilon) \sqrt{e^{\varepsilon}\left(1+X^{2}\right)-1}}{3 a},
\end{aligned}
$$

$$
\widetilde{b}=b+\varepsilon
$$


so that, under this transformation, equation (6) necessarily becomes

$$
\widetilde{X} \widetilde{Y}^{\prime \prime}=\frac{\tilde{b}}{3 a}+\frac{\tilde{b}^{3}}{27 a^{2}}+\left(1+\frac{\tilde{b}^{2}}{3 a}\right) \widetilde{Y}^{\prime}+\tilde{b} \widetilde{Y}^{\prime 2}+a \widetilde{Y}^{\prime 3} .
$$

If we now set $\varepsilon=-b$ in transformations (11)-(13), $\widetilde{b}$ equals zero, and equation (14) reduces to

$$
\tilde{X} \tilde{Y}^{\prime \prime}=\tilde{Y}^{\prime}+a \tilde{Y}^{\prime 3}
$$

Clearly, equation (15) is mapped back to equation (6) via point transformations (11)-(13) with $\varepsilon=-b$, i.e.,

$$
\begin{aligned}
& \tilde{X}=\sqrt{e^{-b}\left(1+X^{2}\right)-1}, \\
& \tilde{Y}=e^{(-b / 2)}\left(Y+\frac{b}{3 a} X\right) .
\end{aligned}
$$

This means that a solution of equation (15) is mapped to a solution of equation (6) via transformation (16).

\section{Reduction of (15) to the Free Particle Equation}

We seek an invertible point transformation between equation (15), which we restate here in the original variables $X$ and $Y$,

$$
X Y^{\prime \prime}=Y^{\prime}+a Y^{\prime 3}
$$

and the free particle equation

$$
W^{\prime \prime}=0, W=W(Z) .
$$

We exploit the equivalence of the symmetry Lie algebras of the two equations to construct the point transformation.

Lie point symmetries admitted by equations (17) and (18) are

$$
\begin{aligned}
& \Phi_{1}=\left(\frac{1}{X}\right) \partial_{X}, \Phi_{2}=\left(\frac{-2 a^{2} Y^{3}}{X}\right) \partial_{X}+\left(3 a Y^{2}+X^{2}\right) \partial_{Y} \\
& \Phi_{3}=\left(\frac{a Y^{2}}{X}+X\right) \partial_{X}, \Phi_{4}=\left(\frac{a Y^{2}}{X}\right) \partial_{X}-Y \partial_{Y}, \\
& \Phi_{5}=\left(\frac{X^{4}-a^{2} Y^{4}}{2 X}\right) \partial_{X}+Y\left(a Y^{2}+X^{2}\right) \partial_{Y}, \\
& \Phi_{6}=\left(\frac{Y}{X}\right) \partial_{X}, \Phi_{7}=\left(\frac{2 a Y}{X}\right) \partial_{X}-\partial_{Y}, \\
& \Phi_{8}=\left(Y X-\frac{a Y^{3}}{X}\right) \partial_{X}+2 Y^{2} \partial_{Y},
\end{aligned}
$$

$$
\begin{aligned}
& \Omega_{1}=\partial_{Z}, \Omega_{2}=\partial_{W}, \Omega_{3}=Z \partial_{Z}, \Omega_{4}=Z \partial_{W}, \Omega_{5}=W \partial_{W} \\
& \Omega_{6}=W \partial_{Z}, \Omega_{7}=Z W \partial_{Z}+W^{2} \partial_{W}, \Omega_{8}=Z^{2} \partial_{Z}-Z W \partial_{W},
\end{aligned}
$$

respectively. The Lie algebras arising from (19) and (20) are equivalent, which means an isomorphism leading to the same commutator table for the two Lie algebras can be found. We may rearrange operators (19) to form a new basis $\left\{\Gamma_{1}, \ldots, \Gamma_{8}\right\}$ such that

$$
\begin{aligned}
{\left[\Gamma_{i}, \Gamma_{j}\right] } & =C_{i j}^{k} \Gamma_{k}, \\
{\left[\Omega_{i}, \Omega_{j}\right] } & =C_{i j}^{k} \Omega_{k},
\end{aligned}
$$

with the same structure constants $\left\{C_{i j}^{k}\right\}$. The following rearrangements provide the desired new basis for the symmetry Lie algebra arising from (19):

$$
\begin{aligned}
& \Gamma_{1}=\gamma \Phi_{6}, \\
& \Gamma_{2}=\gamma \lambda \Phi_{8}, \\
& \Gamma_{3}=\frac{1}{2} \Phi_{3}, \\
& \Gamma_{4}=\lambda \Phi_{4}, \\
& \Gamma_{5}=-\frac{\Phi_{3}}{2}+\Phi_{4}+\gamma \delta \Phi_{8}, \\
& \Gamma_{6}=-\frac{\Phi_{1}}{2 \lambda}+\frac{\delta \gamma \Phi_{6}}{\lambda}, \\
& \Gamma_{7}=-\frac{\delta \Phi_{3}}{2 \lambda}+\frac{2 \delta \Phi_{4}}{\lambda}+\frac{a \Phi_{6}}{2 \gamma \lambda}-\frac{\Phi_{7}}{2 \gamma \lambda}+\frac{\gamma \delta^{2} \Phi_{8}}{\lambda}, \\
& \Gamma_{8}=-\frac{\Phi_{2}}{2 \gamma}+\delta \Phi_{5}+\frac{a \Phi_{8}}{2 \gamma},
\end{aligned}
$$

where $\gamma, \lambda$, and $\delta$ are arbitrary constants with $\lambda \gamma \neq 0$. We now seek a point transformation that maps equation (17) to equation (18) of the form

$$
\begin{array}{r}
Z=\alpha(X, Y), \\
W=\beta(X, Y),
\end{array}
$$

for functions $\alpha$ and $\beta$. According to Chapter 6 of [9], the functions $\alpha$ and $\beta$ must be such that the conditions

$$
\begin{aligned}
\Omega_{i} Z & =\Gamma_{i} \alpha(X, Y), \\
\Omega_{i} W & =\Gamma_{i} \beta(X, Y), \quad i=1, \ldots, 8,
\end{aligned}
$$


are satisfied. The equations in (24) translate into an overdetermined system of sixteen elementary linear PDEs that define the functions $\alpha$ and $\beta$. The equations are easily solved, and we obtain

$$
\begin{gathered}
Z=\alpha(X, Y)=\frac{a Y^{2}+X^{2}}{2 \gamma Y}, \\
W=\beta(X, Y)=\frac{\delta}{\lambda}-\frac{1}{2 \gamma \lambda Y} .
\end{gathered}
$$

The solution of (17) is now recovered from the solution of (18),

$$
W=m Z+c,
$$

through point transformation (25). We obtain

$$
\frac{\delta}{\lambda}-\frac{1}{2 \gamma \lambda Y}=m\left(\frac{a Y^{2}+X^{2}}{2 \gamma Y}\right)+c,
$$

or, equivalently,

$$
a Y^{2}+A Y+X^{2}+B=0
$$

where

$$
\begin{aligned}
& A=\frac{2 \gamma}{m}\left(c-\frac{\delta}{\lambda}\right), \\
& B=\frac{1}{\lambda m} .
\end{aligned}
$$

The solution of generic equation (6) now follows from point transformation (16) and equation (28), stated in terms of the variables $\widetilde{X}$ and $\widetilde{Y}$ of equation (15). It is

$$
a Y^{2}+\frac{2 b}{3} X Y+\left(1+\frac{b^{2}}{9 a}\right) X^{2}+J_{2}\left(Y+\frac{b}{3 a} X\right)+J_{1}=0
$$

where

$$
\begin{aligned}
& J_{1}=e^{b}(B-1)+1, \\
& J_{2}=A e^{(b / 2)} .
\end{aligned}
$$

Equation (30) represents a convenient solution of every linearisable second-order ordinary differential equation; in that, the general solution of every such equation is realisable from equation (30) via a point transformation constructed using only two suitably chosen symmetries of the equation according to Theorem 1 .

\section{Illustrative Examples}

Example 1. The ODE

$$
y^{\prime \prime}+3 y y^{\prime}+y^{3}=0,
$$

called the modified Emdem equation, which arises in a variety of contexts [1], admits the maximal 8 symmetries. Among the admitted symmetries are two noncommuting symmetry generators

$$
\begin{aligned}
& X_{1}=\partial_{x}, \\
& X_{2}=x \partial_{x}-y \partial_{y},
\end{aligned}
$$

which satisfy condition (4) of Theorem 1. Symmetries (33) are reduced to their canonical form (5) via the point transformation

$$
\begin{aligned}
& X=\left(k_{1} / y\right), \\
& Y=x+\left(k_{2} / y\right),
\end{aligned}
$$

where $k_{1}(\neq 0)$ and $k_{2}$ are arbitrary constants (also, see [3]). Under this, point transformation equation (32) is reduced to equation (6) with $a=-k_{1}^{2}$ and $b=3 k_{1}\left(k_{2}+1\right)$, and the solution in (30) is transformed into

$$
y=\frac{2 k_{1}^{2} x-J_{2}}{k_{1}^{2} x^{2}-J_{2} x-J_{1}},
$$

which is the desired general solution of (32).

Example 2. Consider ODE No. 6.180 of Kamke [15]:

$$
y^{\prime \prime}(y-1)^{2} x^{2}-2 y^{\prime 2} x^{2}-y^{\prime}(y-1) x-2 y(y-1)^{2}=0 .
$$

This equation satisfies the conditions in Item 3 of Theorem 1 and therefore admits the maximal 8 Lie point symmetries, two of which are

$$
\begin{aligned}
& X_{1}=\left(\frac{y-2}{y-1}\right) \partial_{x}+\frac{2 y}{x} \partial_{y} . \\
& X_{2}=x \partial_{x} .
\end{aligned}
$$

These infinitesimal symmetries satisfy condition (4) from Theorem 1 and are reduceable to their canonical forms (5) via the point transformation

$$
\begin{aligned}
& X=\frac{k_{2} x \sqrt{1-y}}{y}, \\
& Y=\left(\frac{k_{1} \sqrt{1-y}}{y}+1-\frac{1}{y}\right) x,
\end{aligned}
$$

where $k_{1}$ and $k_{2}(\neq 0)$ are arbitrary constants. Under this, transformation equation (36) is reduced to equation (6), with $a=-k_{2}^{2}$ and $b=3 k_{1} k_{2}$. The general solution of (36) now follows easily from equation (30) written in terms of $x$ and $y$ via point transformation (38), with $a$ and $b$ set to $-k_{2}^{2}$ and $3 k_{1} k_{2}$, respectively. We obtain

$$
y=\frac{x\left(k_{2}^{2} x-J_{2}\right)}{k_{2}^{2} x^{2}-J_{2} x-J_{1}} .
$$

Example 3. Consider the linearisable equation in p.100 of [11]: 


$$
x y^{\prime \prime}=2\left(y^{\prime}\right)^{2}+y^{\prime}+y^{2} .
$$

The symmetries

$$
\begin{aligned}
& X_{1}=\left(1-\frac{1}{x y}\right) \partial_{x}+\frac{y}{x} \partial_{y}, \\
& X_{2}=\left(x^{2}-\frac{x}{y}\right) \partial_{x}+(2-3 x y) \partial_{y},
\end{aligned}
$$

are admitted by (40) and satisfy condition (14). The point transformation that maps symmetries (41) to their canonical form (5) is

$$
\begin{aligned}
& X=\sqrt{\frac{2 x y-1}{y^{2}}-1,} \\
& Y=\frac{x y-1}{y} .
\end{aligned}
$$

This transformation also reduces equation (40) to equation (6), with $a=1$ and $b=0$. Writing solution (31) in terms of $x$ and $y$ via point transformation (42) and setting $a=1$ and $b=0$, we obtain the solution to (40):

$$
y=\frac{J_{2}}{x^{2}+J_{2} x+J_{1}-1} .
$$

\section{Concluding Remarks}

There are many characterisations of linearisable secondorder ODEs. One important characterisation is that every such equation is reduceable to a generic second-order ODE, equation (6), via a point transformation constructed from two suitably chosen symmetries. We have used the expanded Lie group approach to simplify equation (6) significantly via a point transformation constructed to set the parameter $b$ in equation (6) to zero. The reduced equation is subsequently mapped to the free particle equation, $y^{\prime \prime}=0$, via another invertible point transformation constructed by "aligning" the respective symmetries of the two equations. The constructed point transformations are used in succession to obtain the general solution to equation (6), which is the desired proxy solution for all linearisable second-order differential equations. This allows construction of solutions of all equations in this class algorithmically using only two suitably chosen symmetries of the equation. We have illustrated the solution routine with three examples.

\section{Data Availability}

No data were used to support this study.

\section{Conflicts of Interest}

The author declares that there are no conflicts of interest regarding the publication of this paper.

\section{Acknowledgments}

The author would like to thank the Directorate of Research Development and Innovation of Walter Sisulu University for continued financial support.

\section{References}

[1] M. Senthilvelan, V. K. Chandrasekar, and R. Mohanasubha, "Symmetries of nonlinear ordinary differential equations: the modified emden equation as a case study," Pramana-Journal of Physics, vol. 85, no. 5, pp. 755-787, 2015.

[2] M. Safdar, S. Ali, and F. M. Mahomed, "Linearization of systems of four second-order ordinary differential equations," Pramana-Journal of Physics, vol. 73, no. 3, pp. 581-594, 2011.

[3] F. M. Mahomed, "Symmetry group classification of ordinary differential equations: survey of some results," Mathematical Methods in the Applied Sciences, vol. 30, pp. 1995-2012, 2007.

[4] F. Schwarz, Algorithmic Lie Theory for Solving Ordinary Differential Equations, Chapman \& Hall/CRC, Boca Raton, FL, USA, 2008.

[5] M. T. Mustafa and A. Y. Al-Dweik, "Mara'beh on the linearization of second-order ordinary differential equations to the laguerre form via generalized sundman transformations," Symmetry, Integrability and Geometry: Methods and Applications SIGMA, vol. 9, p. 10, 2013.

[6] W. Nakpim and S. V. Meleshko, "Linearization of secondorder ordinary differential equations by generalized sundman transformations," SIGMA, vol. 6, no. 51, p. 11, 2010.

[7] W. Nakpim, "Third-order ordinary differential equations equivalent to linear second-order ordinary differential equations via tangent transformations," Journal of Symbolic Computation, vol. 77, pp. 63-77, 2016.

[8] G. Baumann, Symmetry Analysis of Differential Equations with Mathematica, Springer, Berlin, Germany, 2000.

[9] G. W. Bluman and S. Kumei, Symmetries and Differential Equations, Springer-Verlag, Berlin, Germany, 1989.

[10] B. J. Cantwell, Introduction to Symmetry Analysis, Cambridge University Press, Cambridge, UK, 2002.

[11] P. E. Hydon, Symmetry Methods for Differential Equations: A Beginner's Guide, Cambridge University Press, Cambridge, UK, 2000

[12] L. V. Ovsiannikov, Group Analysis of Differential Equations, Academic Press, Cambridge, MA, USA, 1982.

[13] P. J. Olver, Applications of Lie Groups to Differential Equations, Springer-Verlag, Berlin, Germany, 1993.

[14] G. I. Burde, "Expanded Lie group transformations and similarity reductions of differential equations," Proceedings of Institute of Mathematics of NAS of Ukraine, vol. 43, no. 1, pp. 93-101, 2002.

[15] E. Kamke, Differentialgleichungen: Lösungsmethoden und Lösungen, I, Gewöhnliche Differential-Gleichungen, B. G. Teubner, Leipzig, Germany, 1977. 